\title{
TWO INNOVATIVE DEVICES FOR THE TEACHING OF ASTRONOMY
}

\author{
Roland Szostak \\ Institut für Didaktik der Physik, Universität Münster, Fachbereich Physik, \\ Wilhelm-Klemm-Str. 10, D-4000 Münster, Federal Republic of Germany
}

The teaching of astronomy in schools should start by explaining the most elementary phenomena, which are a part of everyone's daily experience. Before proceeding to advanced topics, teachers of astronomy in school have to make sure that these very basic phenomena are fully understood. Since many programs have a gap at the elementary level, many people have foggy notions or disconcertingly wrong ideas of elementary matters. The seasons are a well-known case of a badlyunderstood phenomenon even for highly educated people. Still these people are honest and should not be laughed at. They do not understand the basic concepts of our contemporary scientific world, simply because these topics have not been taught properly. This problem raises a challenge for developing better ways of teaching astronomy. In order to show that there are specific new solutions to this challenge, I am presenting two innovative devices, which have been developed at the Universität Münster. The first example shows how the seasons may be successfully explained with a physical model that changes color when heated. The other example refers to the visibility of the stars and their disappearance in the daytime.

\section{Thermal Method for Describing Seasons}

The standard method for teaching these basic phenomena uses simple geometric optics. By depicting the rays of light from the sun, it is easy to recognize the illuminated parts of the Earth and moon. So, for the phenomena of night and day, and the phases of the moon, we do not feel that it is necessary to visualize the related rotational and orbital movements. But the situation is different when explaining the seasons. There, the angle of incidence and the duration of irradiation have to be considered for each place on the rotating Earth. To keep it simple, we focus our analysis on the solstice positions. For these two positions, a cut through the meridional plane allows us to see that the rays of the sun are steeper at the summer solstice than at the winter solstice. Although this analysis is only static, it affords some skill in geometry. Trying to include motions as well would involve superimposed rotational transformations, whose details become very sophisticated. Geometric optics would then no longer be sufficient for the model.

But we may ask whether the seasons should be explained in this way. Children identify winter with low temperatures, with snow and ice, and summer with high temperatures, but they are not so much aware of the changing position of the sun in the sky. So it would be much better to use a temperature effect for explaining the seasons. I report such an approach here. 
Our technique uses a thermally sensitive material $\left(\mathrm{Ag}_{2} \mathrm{HgI}_{4}\right)$ that looks yellow at room temperature and turns reversibly red at about $40^{\circ} \mathrm{C}$. This material can be obtained from suppliers such as the Merck Company in the form of a salt of tiny crystals in a glass container. These crystals have to be suspended in a colorless transparent paint to fix them to a surface. I have coated a sphere of styrofoam with this material, and exposed this model globe to a heater or a spotlight. The most intensively irradiated parts of the sphere turn red. A rotating sphere irradiated from its equatorial plane shows a red ring on its equatorial zone. Children in a classroom accept this as being obvious, and are fascinated by this play of colors. Assigning the color red to the hot areas seems intuitively natural.

How does this method work with respect to our seasons? Where we live, the sphere should be yellow in winter and red in summer. In a first naive attempt, this effect would be achieved by putting the rotating sphere somewhat closer to or further from the heater. Then the red ring would become broader or narrower. But as the ring would remain symmetric with respect to the equator, and the some seasons would occur simultaneously in the northern and southern hemisphere, this turns out to be a wrong idea. In order to get opposite seasons in the two hemispheres, the red ring has to shift somewhat towards the north or south, oscillating around its symmetric equatorial position. But this cannot be achieved by changing the distance to the sun. Children in a classroom find out, simply by playing, that the red ring is shifted by tilting the axis. This visual experience promotes a more effective understanding.

Now one is ready to carry the sphere around the heater to observe how the hot zone is shifted by the orbital path of the Earth. It becomes evident that, without the inclination of the axis, there would be no seasons. The orbit leads to a year with seasons because the axis is tilted. In my experience, this procedure works very satisfactorily in classes with children down to the age of nine. The children even make the statement that the orientation of the axis in space must remain constant. The idea of checking this orientation with the poler star seems to suggest itself here.

A crucial difference between the common optical method and this new thermal method is the irradiated heat is stored, whereas the incident light is available instantaneously only. By virtue of this storage, the sphere displays the temperature distribution on the whole globe, independently of the day-night position. This averaging over the short day-night period leaves just the smoothed behavior of the seasonal temperature variation, which is not accessible by the optical method.

Let me add some technical remarks: The model sphere needs to be heated up for some time (a minute or so) before it changes its color. Similarly, it holds the stored heat for some time, so that the temperature distribution can be seen by everyone in the classroom at leisure. This thermal inertia offers an additional interesting feature: If the rotating sphere moves around the heater slowly, like the Earth in its orbital path, then the displayed temperature distribution shows a characteristic time lag, just as lowest and highest temperature occur not at the solstices but several weeks later. For proper display, the orbital speed has to be adjusted to the thermal reaction time of the model sphere. 

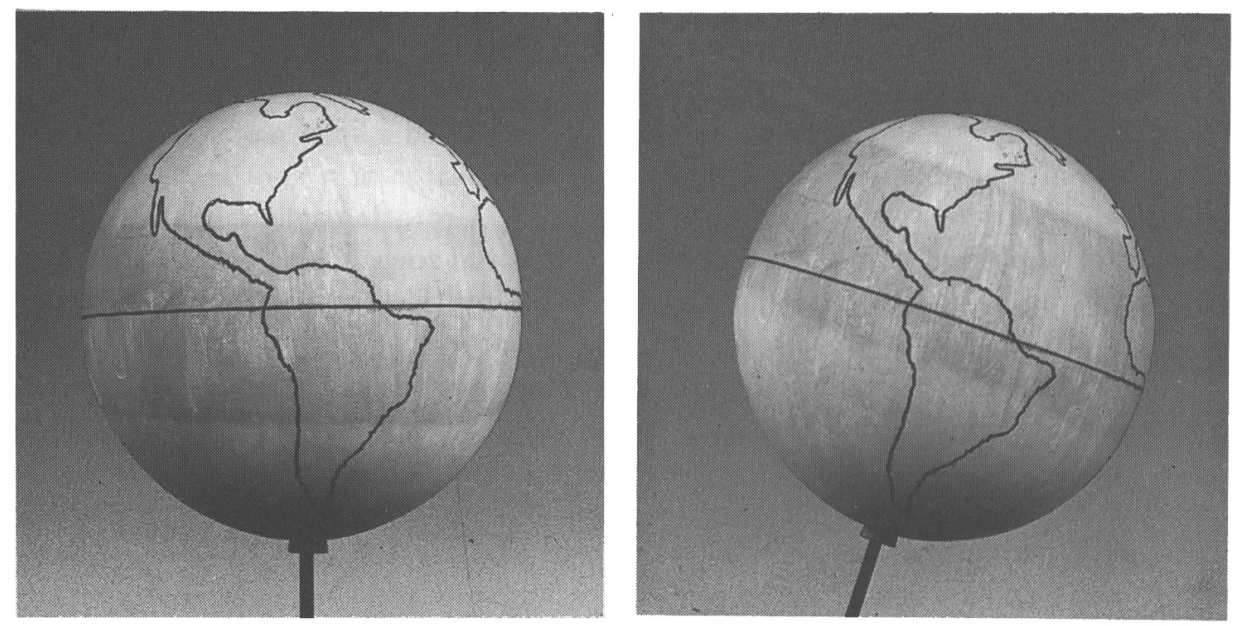

Figure 1. The chemical on the ball turns from yellow to red (lighter to darker on this black-and-white photograph) when warmed. a) The appearance of the ball if the axis were upright and so with the sun shining directly down on the equator. b) The appearance of the ball for the northern summer solstice, which the Earth's axis is tilted toward the sun. (The sun is off to the right in both photographs.)

This reaction time is determined by the specific heat capacity and the thermal conductance of the material. Both of these values should be as low as possible for practical purposes, in order to reduce the power of the heater and to get a reaction quickly. Styrofoam turned out to be a suitable material. To lower these values further, we coated the sphere in some cases with a velvet layer about $0.5-1.0$ $\mathrm{mm}$ thickness before applying the temperature-sensitive paint. This velvet consists of short pieces of organic fibers which are fixed to the surface electrostatically by a standard industrial procedure. Generally, the styrofoam has to be very homogeneous in order to keep the areas where the color is changing from looking somewhat stained.

\section{Star Charts}

Let me now deal with the starfinders that are used by amateurs. In order to familiarize students with this tool, we made a planisphere for overhead projection in the classroom. Instead of using a transparent foil, we took an opaque material with little holes for the stars. In order to make this stellar sky rotate around the pole star, the star disk is fixed by a snap onto an underlying transparent sheet that has an opaque cover for the parts outside of the horizon, so that the stars can be seen entering and leaving this horizon area.

Compared to other ways of visualization, this projected stellar sky is surprisingly impressive in a darkened classroom. It may initiate students into how attractive it must be to observe the stars in nature. Although such an experience can be 
better supplied by a planetarium, the advantage of this cheap overhead projection is the individual interaction between students and the teacher that is not available in the planetarium during a show. As a result, the teacher can secure the knowledge of constellations like the Big Dipper, Orion, etc. The teacher can select these stars very simply with a sheet of paper having a suitable aperture to prevent all other stars from being projected. Thus the constellation can be seen and memorized very effectively. Such a step is not trivial, because most constellations are barely known. Further, it is worthwhile to show how the Big Dipper changes its orientation hour by hour, thus making students aware of the rotation of the earth.

When using these planispheres, one very soon arrives at the question of the visibility of the stars in the daytime. At this point, one can make clear that the stars are still there, and that they are not visible because the sun is flooding the atmosphere with light. It helps to remind students of the fact that some bright stars or planets can be seen in the early morning or when dusk is falling. Children become aware of this effect when they are shown how the stars emerge from twilight. This process is much better visualized on a quicker time scale. We simulate sunrise and sunset on an overhead projector in the following way: we take two polarizing sheets about $30 \mathrm{~cm}$ by $30 \mathrm{~cm}$ covering the projector's flat surface. One of these two foils has a pattern of small holes drilled at the positions of the stars. When the polarization axes of these foils are parallel, the projector's light passes through and the stars are almost invisible. But when the second foil, which contains no holes, is turned into the crossed position, the "sky" slowly darkens. Meanwhile, the stars emerge from twilight as if dusk is falling; they finally appear as brilliant spots on the dark background. This procedure can be very impressive, bring the children in the classroom into contact with what can be observed in nature with some patience.

Some additional optical effects can add further intellectual content. One such technique allows us to introduce extra motions of celestial bodies into this projected stellar sky. We use the fact that light passes again through the crossed filters when a quarter-wave plate is inserted between them, transforming the linearly polarized light from the first polarizing sheet into circularly polarized light. By shaping this quarter-wave plate like the silhouette of the moon, one can have a bright moon projected together with the other stars on the dark background. But this moon can now be moved by hand through the pattern of the other projected stars. In the same way, of course, a planet instead of the moon can be introduced into the projected stellar sky. So the apparent motions of the planets through the stellar sky, including their retrograde loops, can be shown and discussed very specifically and individually in the classroom.

A second technique allow us to introduce colors into scenery by using the above optical effect's wavelength dependence. Not all the light being turned into the circularly polarized mode can pass through the second filter; only light within a certain window of wavelengths does so. As a result, this projected light looks colored. It is fortunate that foils with a suitable dichroic behavior are extremely cheap and readily available. Clear plastic used for wrapping bunches of flowers or candy boxes and even clear stickytape works perfectly. By simply inserting such 
colorless foils between the two polarizing filters, the projected dark sky turns a color; meanwhile, the stars stay bright and colorless. By turning the foil and the second polarizing filter into adequate orientations, the color itself and its saturation can be chosen at will. In this way, the night-black sky can be turned into a deeply saturated blue and continuously into a light blue as the stars stay unchanged and so fade away in the rising light. In this simulation of the morning hours, the blue can also be changed into red or other colors. Further, the moon, when rising above horizon, may be projected in orange and can be gradually turned into white.

All the above can be done in a classroom with a few foils on an overhead projector. The process is very effective and allows individual discussion of astronomical phenomena between children and teacher. Although the process allows some effects of a planetarium into the classroom, it is not meant to be a substitute for a planetarium. But it can be an effective preparation for a planetarium visit, because several phenomena that are shown in the planetarium will be better understood by the children after such prior discussion.

\section{Discussion}

G.L. Verschuur: This contribution highlights what I believe is an important point regarding astronomy courses in the U.S.A. The great majority of students are in the class to fulfill their science requirement. The goal is to give them a sense of what science is about, how scientists think and how they approach problems. The fact that we use astronomy as an example is almost secondary (although it may be heretical to suggest that here). Thus we do not have to teach all of astronomy. We do want to show how the scientific method is used to answer questions regarding the nature of phenomena such as the seasons, as you have so elegantly demonstrated.

J.-C. Pecker: One should realize that, at the university level, there are two ways to teach astronomy; one is to do it as a "natural science," using physics of course, but as a tool; another one is to do it as a series of applications of physical concepts. The latter is becoming quite a fashion in some places, and I would object to it. The remark by Verschuur might lead to similar difficulties, i.e., losing the unity of astronomy seen as a "natural science." 